\title{
Water Intake and Ion Exchange in PEDOT:Tos Films upon Cyclic Voltammetry: Experimental and Molecular Dynamics Investigation
}

Najmeh Delavari, Johannes Gladisch, Ioannis Petsagkourakis, Xianjie Liu, Mohsen Modarresi, Mats Fahlman, Eleni Stavrinidou, Mathieu Linares and Igor Zozoulenko

The self-archived postprint version of this journal article is available at Linköping University Institutional Repository (DiVA):

http://urn.kb.se/resolve?urn=urn:nbn:se:liu:diva-178503

N.B.: When citing this work, cite the original publication.

Delavari, N., Gladisch, J., Petsagkourakis, I., Liu, X., Modarresi, M., Fahlman, M., Stavrinidou, E., Linares, M., Zozoulenko, I., (2021), Water Intake and Ion Exchange in PEDOT:Tos Films upon Cyclic Voltammetry: Experimental and Molecular Dynamics Investigation, Macromolecules, 54(13), 65526562. https://doi.org/10.1021/acs.macromol.1c00723

Original publication available at:

https://doi.org/10.1021/acs.macromol.1c00723

Copyright: American Chemical Society

http://pubs.acs.org/ 


\section{Water Intake and Ion Exchange in PEDOT:Tos Film upon Cyclic Voltammetry: Experimental and Molecular Dynamics Investigation}

Najmeh Delavari, ${ }^{1}$ Johannes Gladisch, ${ }^{1}$ Ioannis Petsagkourakis, ${ }^{1}$ Xianjie Liu, ${ }^{1}$ Mohsen Modarresi, ${ }^{2}$

Mats Fahlman, ${ }^{1}$ Eleni Stavrinidou, ${ }^{1}$ Mathieu Linares, ${ }^{1,3,4}$ Igor Zozoulenko $^{1}$

${ }^{1}$ Laboratory of Organic Electronics (LOE), Department of Science and Technology (ITN), Campus Norrköping, Linköping University, SE-60174 Norrköping, Sweden

${ }^{2}$ Department of Physics, Ferdowsi University of Mashhad, Mashhad, Iran

${ }^{3}$ Group of Scientific Visualization, Department of Science and Technology (ITN), Campus Norrköping,

Linköping University, SE-60174 Norrköping, Sweden

${ }^{4}$ Swedish e-Science Center (SeRC), Linköping University, SE-581 83 Linköping, Sweden

\section{Graphical Abstract}
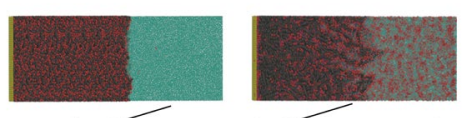

Quartz Crystal Microbalance
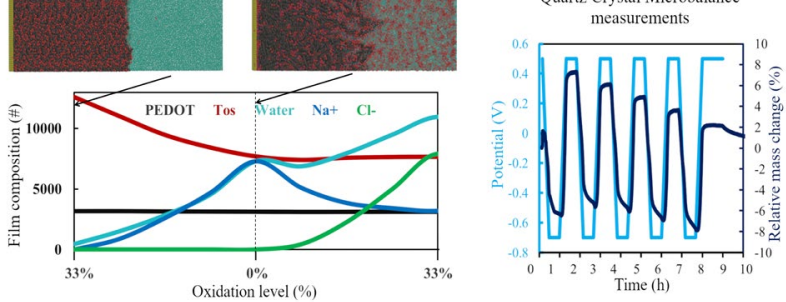


\section{Abstract}

Conductive polymer PEDOT:Tos (3,4- ethylenedioxythiophene doped with molecular tosylate) gained a considerable attention in various devices for bioelectronic applications, such as organic transistors and sensors. Many of these devices function upon oxidation/reduction processes in contact with aqueous electrolytes. So far, a theoretical insight into morphological changes, ion injection and water intake during these processes was rather limited. In the present work, we combined experiments and molecular dynamics simulations to study the water intake, swelling and exchange of ions in PEDOT:Tos film during the cyclic voltammetry. We showed that the film underwent significant changes in morphology and mass during the redox processes. We observed both experimentally and in simulations that the film lost its mass during reduction as tosylate and $\mathrm{Na}$ was expelled and gained mass during oxidation mainly due to the uptake of anions, i.e. tosylate and $\mathrm{Cl}$. The results were in line with UV-VIS-NIR absorption measurements, and X-ray photoelectron spectroscopy (XPS) measurements, which revealed that during red-ox process a portion of Tos was replaced by $\mathrm{Cl}$ - as the counter-ion for PEDOT. Also, the relative mass change between the most oxidized and reduced states was $\sim 10-14 \%$ according to both experiment and simulations. We detected an overall material loss of the film during voltammetry cycles indicating that a portion of the material leaving the film during reduction did not return to the film during the consecutive oxidation. Our combined experimental/simulation study unravelled the underlying molecular processes in the PEDOT:Tos film upon the redox process, providing the essential understanding needed to improve and assess the performance of bioelectronic devices.

Keywords: conductive polymers, PEDOT:Tos film, water intake, ion exchange, cyclic voltammetry, MD simulations, Quartz Crystal Microbalance with dissipation monitoring, XPS 


\section{Introduction}

Over the past two decades, organic materials and in particular conducting polymers have become an indispensable part of many electronic and bioelectronic devices. Polymer-based organic electronic devices are extensively studied and explored; they are low-cost, flexible, printable and solution processable. Among all conjugated polymers, poly(3,4ethylenedioxythiophene) (best known as PEDOT) is one of the most studied ${ }^{1-5}$ and is particularly known for being highly conductive with the electric conductivity exceeding 1000 $\mathrm{S} \mathrm{cm}^{-1} .{ }^{6-9}$ PEDOT is rather transparent in the visible spectrum when oxidized and changes to dark blue when reduced. ${ }^{10}$ PEDOT can also act as a mixed conductor since it can present both electronic and ionic conductivity. ${ }^{9,11,12}$ All these unique features along with its considerable stability in air and upon thermal changes make PEDOT a promising material for an extensive range of applications in polymer electronic devices such as organic electrochemical transistors (OECT), ${ }^{13}$ smart surfaces for cell control, ${ }^{14-17}$ thermoelectric devices, ${ }^{7}$ electrochromic displays, ${ }^{18}$ source and drain electrodes, ${ }^{19}$ electrodes in contact with neuronal systems, ${ }^{20}$ drug delivery devices ${ }^{21}$ (i.e., organic electronic ion pumps), supercapacitors, ${ }^{22}$ organic light emitting diodes, ${ }^{23}$ organic field effect transistors, ${ }^{24}$ and other applications.

Oxidative polymerization in the presence of negative counterions, categorizes PEDOT as a ptype organic semiconductor. During the polymerization process, electronic charges on PEDOT are compensated by polymeric counterions such as negatively charged polystyrene sulfonate (PSS) or molecular counterions such as tosylate (Tos). A recent study has provided the essential theoretical insights to understand the thermodynamics and kinetics of PEDOT:Tos oxidative polymerization and successfully revealed why it is typically oxidized up to $33 \%$. ${ }^{25}$ It is commonly admitted that the conducting properties of PEDOT originate from the transport of holes (positive charge carriers) through the conjugated bonds in the backbone of the polymer 
chains. ${ }^{26-29}$ PEDOT:Tos has attracted lots of attention because it is highly conductive, showing semi-metallic behavior, good stability ${ }^{30}$, and boasts interesting thermoelectric properties ${ }^{26,31}$, ${ }^{32}$ Furthermore PEDOT:Tos was explored for state-of-the-art bioelectronic applications where electronic devices are in close contact with a biological environment or living organs (usually an electrolytic medium). ${ }^{14-17,33}$

Several experimental studies were conducted to investigate the underlying morphology and ion diffusion in PEDOT:Tos. ${ }^{7,8,32,34-36}$ Crystallization of PEDOT:Tos in aqueous solution was studied using all atomistic molecular dynamics simulations, and the relation between the morphology and the mobility, the effect of the substrate and the ionic diffusion in PEDOT:Tos were modelled theoretically. ${ }^{37,38}$

During last years, water intake, swelling and ion injection in conducting polymer has attracted great attention in the literature because these processes change the electrical and optical properties of the material and correlate significantly with the device performance. Understanding those changes is crucial to guide the design of future materials with improved performances. Typically, water intake and the ion penetration are investigated under cyclic voltammetry condition when the ions enter or leave the polymeric film to compensate for the charges that are inserted to or released from the film during oxidation or reduction. This phenomenon may lead to adsorption of ions on the surface of the polymer film and swelling of the film. A powerful technique to investigate morphological changes in the polymer is the Quartz Crystal Microbalance with dissipation monitoring (QCMD) allowing to detect tiny mass changes of the polymer film during cyclic voltammetry. ${ }^{39}$ A combined electrochemical QCM studies were performed by Wieland et al. to monitor the water uptake and conductivity changes in PEDOT:PSS films upon changes in humidity. ${ }^{40}$ Also, this technique was used by Savva et al. to monitor the movement of $\mathrm{Na}+$ ions and water between PEDOT:PSS and electrolyte. ${ }^{41}$ Note that the origin of the swelling of PEDOT:PSS films was recently unravelled by the 
molecular dynamics modelling of Modarresi et al. ${ }^{42}$ The QCMD technique was recently used for glycol derivates of polythiophenes to monitor of the phase transition of the polymer which took place under cyclic voltammetry. ${ }^{43,44}$ The ion injection and water intake were a subject of a recent study by Gladisch et al. who reported reversible electronic solid-gel switching and gigantic swelling of a conductive polymer. ${ }^{45}$ Savva et al. studied operation of a polymer organic electrochemical transistor (OECT) where a relationship was found between the OECT performance and the balanced mixed conduction of the polymer. ${ }^{44}$

The studies mentioned above focussed mostly on PEDOT:PSS and polymers with polythiophene-based backbone and ethylene glycol side chains, whereas PEDOT:Tos has so far received much less attention. Also, most reported studies have been experimental ones, where, therefore, a theoretical insight into morphological changes was rather limited. In the present paper, we report Molecular Dynamic (MD) simulations combined with experimental studies (X-ray photoelectron spectroscopy (XPS), electrochemical Quartz Crystal Microbalance (eQCMD), and UV-VIS-NIR absorption spectroscopy) to gain a theoretical insight on the water intake, polymer swelling and ion exchange in PEDOT:Tos film during cyclic voltammetry. MD is a powerful tool making possible to obtain detailed morphological information that is not accessible via conventional experimental techniques. Because of this it is sometimes called “computational microscopy". Our simulations not only quantitatively reproduce the experimental results, but allow us to monitor structural changes in the polymer film at the nanoscale thereby providing molecular understanding of the evolution of the morphology and composition in the system at hand.

\section{Experimental setup and computational modelling}




\section{II.A. Experimental section (film preparation and EQCM measurements)}

II.A.a. In situ chemical polymerization of PEDOT Tosylate films

3,4-ethylenedioxythiophene (EDOT), pyridine, dimethylsulfoxide (DMSO), n-butanol, ethanol, were purchased from Sigma-Aldrich. The iron toluenesulfonate solution (Clevios CB54) was purchased from Heraeus. Silicon wafers were purchased from Silicon Valley Microelectronics Inc. In-situ wet chemical polymerization was used to produce the thin PEDOT:Tos films. ${ }^{46}$ Initially, the iron toluenesulfonate solution was diluted at $40 \mathrm{wt}-\%$ in iron tosylate with n-butanol and stirred overnight at room temperature. Then, the additives, pyridine and DMSO, were both added at $3 \mathrm{v}-\%$ concentration. The resulted solution (solution 1) was stirring for 12 hours at room temperature before use. For the polymerization, the monomer EDOT was added in a ratio of $3.3 / 100 \mu$ of solution 1 . This dispersion was stirred for $30 \mathrm{~s}$ followed by spincoating on the silicon wafer $(1500 \mathrm{rpm}, 30 \mathrm{~s}, 800 \mathrm{rpm} / \mathrm{s})$. The films $/ \mathrm{Si}$ were annealed at $100{ }^{\circ} \mathrm{C}$ for 15 min to initiate the polymerization and then they were washed in $\mathrm{n}$ butanol, $5 \mathrm{~min}$, in order to remove excess oxidant. After 5 mins and while in the n-butanol bath, the PEDOT films were delaminated with tweezers and transferred to the QCM substrates. The PEDOT:Tos/QCM samples were carefully dried with dry air. Then they were introduced into a two-step bathing process in n-butanol and ethanol, 5 min each, in order to further clean the PEDOT systems. Finally, the samples were dried with dry air.

\section{II.A.b. e-QCMD experiments}

Quartz Crystal Microbalance with dissipation monitoring (QCMD) is an experimental technique that can be used to detect tiny mass changes on the surfaces of the quartzes based on changes in the quartz's resonance frequency. ${ }^{39}$ The dissipation monitoring hereby enables the monitoring of complex systems in water that exhibit viscoelastic behavior, like polymeric films containing water. ${ }^{44,47}$ With a combination of QCMD and electrochemical 
techniques (e-QCMD), the responses of the material on the quartzes to electrochemical triggers and vice versa can be measured. The technique has been applied for a wide range of conjugated polymers, both with attention to the influence of structural properties on ionic-electronic properties as well as the influence of electrochemical triggers on structural properties. ${ }^{40,41,43-45 \text {, }}$ 48, 49 For instance, Stavrinidou et al. used electrochemical quartz crystal microbalance to gain indicators of ion movement in PEDOT:Tos composites. ${ }^{36}$

Prior to the experiment the electrolyte $(0.01 \mathrm{M}$ aqueous, neutral $\mathrm{pH} \mathrm{NaCl})$ and the quartzes (QSense QSX301) with the PEDOT:Toslyate films were purged with Nitrogen for $2 \mathrm{~h}$. The quartzes where then mounted in the QSense electrochemical cell for the quartz crystal microbalance with dissipation device (QCMD, Qsense Analyzer). The e-QCMD was controlled with the QSoft 401 software. The electrochemical experiments were performed in a 3-electrode setup with an $\mathrm{Ag} / \mathrm{AgCl}$ reference electrode (Dri-REFTM, World precision instruments Inc., 3M $\mathrm{KCl}$ ) and a Pt counter electrode integrated into the e-QCMD chamber. The experiments were controlled with a Metrohm $\mu$ Autolab Type III and NOVA 2 software. Potentials were ramped with $1 \mathrm{mV} / \mathrm{s}$ between 0.5 and $-0.7 \mathrm{~V}$. At 0.5 and $-0.7 \mathrm{~V}$ respectively, potentiostatic phases were introduced to ensure, and gather additional information regarding, completion of oxidation and reduction processes. To investigate the impact of oxygen reduction reaction on the mass changes, also independent investigations with $\mathrm{CVs}$ at different scan rates were performed. After the experiments, the related e-QCMD measurements were stitched together to enable frequency to mass conversion with QSense Dfind software. The frequency to mass conversion was done with the Smartfit viscoelastic modelling function of QSense Dfind including overtones 3 to 11 . The chi square based fit quality factor was equal to 0.57 . Therefore, we also converted the frequency changes to mass changes using the Sauerbrey equation. The relative mass change as calculated with the two methods was very similar as shown in Fig. S1.

II.A.c PEDOT:TOS films preparation for UV-VIS-NIR absorption spectroscopy and XPS 
The PEDOT:TOS films for XPS and UV-VIS-NIR absorption spectroscopy were prepared with the same protocol as the films for QCM studies but on FTO substrates. PEDOT:TOS was electrochemically reduced or oxidized following the same protocol as in the e-QCM experiments but performed in an open 3-electrode electrochemical cell with platinum counter electrode, $\mathrm{Ag} / \mathrm{AgCl}$ reference electrode and $0.01 \mathrm{M} \mathrm{NaCl}$ electrolyte. To electrochemically reduce the films, we applied a potential sweep from $+0.5 \mathrm{~V}$ to $-0.7 \mathrm{~V}$ at $1 \mathrm{mV} / \mathrm{s}$ and then the potential was kept constant at $-0.7 \mathrm{~V}$ for $30 \mathrm{~min}$ in order the ensure the completion of the reduction process. To electrochemically oxidize the film a potential sweep from $+0.5 \mathrm{~V}$ to $0.7 \mathrm{~V}$ at $1 \mathrm{mV} / \mathrm{s}$ was first applied, then the potential was kept constant at $-0.7 \mathrm{~V}$ for $30 \mathrm{~min}$, then a potential sweep from -0.7 to $+0.5 \mathrm{~V}$ at $1 \mathrm{mV} / \mathrm{s}$ applied and finally the potential was kept constant at $+0.5 \mathrm{~V}$ for $30 \mathrm{~min}$. Once the electrochemical procedure was completed, the samples were disconnected from the set-up, gently dried with nitrogen and then characterized with UVVIS-NIR absorption spectroscopy and XPS.

\section{II.A.d. $U V$-Vis-NIR spectroscopy}

The spectroscopic experiments were performed on the pristine and the electrochemically reduced or oxidized PEDOT:Tos films with a Perkin Elmer Lambda 900 spectrometer between $250 \mathrm{~nm}$ and $3000 \mathrm{~nm}$ with $1 \mathrm{~nm}$ step.

\section{II.A.e. XPS experiments}

XPS (X-ray photoelectron spectroscopy) measurements was performed in Scienta ESCA 200 system under the base pressure of 2E-10 mbar with SES 200 electron analyzer and a monochromatic Al Ka x-ray source $(\mathrm{hu}=1486.6 \mathrm{eV})$. All XPS spectra were collected at normal emission and at room temperature. The spectrometer was calibrated by a sputter-cleaned $\mathrm{Au}$ film with the Fermi level at $0 \mathrm{eV}$ and $\mathrm{Au} 4 \mathrm{f} 7 / 2$ peak at $84.0 \mathrm{eV}$ with its full width at half maximum being $0.65 \mathrm{eV}$. 


\section{II.B. Computational methodology}

In the present work Coarse-Grained (CG) MARTINI Molecular Dynamics (MD) simulations ${ }^{50}$ were performed using the GROMACS software package. ${ }^{51-53}$ In the CG MD simulations the atoms are grouped in specific beads to cut both complexity and computational cost down, making CG extremely useful for simulating larger systems at longer time scales.

\section{II.B.a. Film preparation}

To prepare a PEDOT film we use a procedure mimicking an experimental technique of the thin film formation during water evaporation. ${ }^{38,54}$ We start with a system consisting of randomly distributed PEDOT chains, tosylate counterions and polarizable water molecules ${ }^{55}$ under $3 \mathrm{D}$ periodic boundary placed in a computational cubic box $20 \times 20 \times 20 \mathrm{~nm}^{3}$. We use a Martini coarse-grained model for PEDOT as developed in the reference ${ }^{56}$. Figure 1 shows the atomic structure and the CG beads for PEDOT chain and tosylate. CG-PEDOT contains MARTINI beads, namely, SNda, SC1, SC2 and a virtual site where the charge on each monomer is defined. The dioxy group in PEDOT structure is grouped as SNda, the sulfur atom as SC2 bead and the four remaining carbons are grouped as SC1 beads. ${ }^{50}$ The structure of the tosylate is depicted in Figure $1 \mathrm{~b}$ where its CG beads are shown. This is based on the model developed in reference ${ }^{57}$. The tosylate model consists of SCY and STY beads and the charged Qa bead represents the charged sulfonate group. ${ }^{56,58,59}$ 
a) PEDOT-12

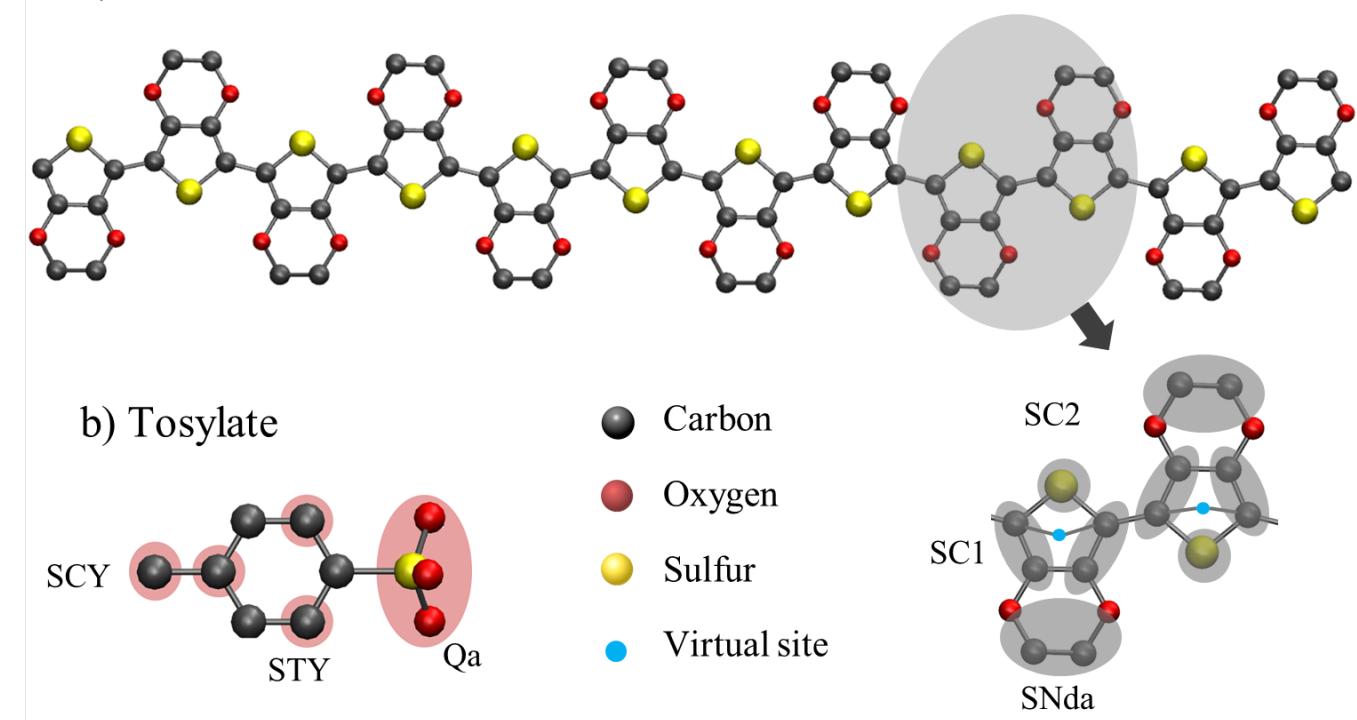

Figure 1. The atomic structure and the corresponding MARTINI model for (a) PEDOT-12 and (b) tosylate.

The initial simulation box contains 400 PEDOT chains of length $\mathrm{N}=12$ monomers, and 51652 water molecules. In the experiment the pristine (i.e. as polymerized) PEDOT typically has a $33 \%$ oxidation level; ${ }^{25}$ hence we set the total charge on the PEDOT chain with $\mathrm{N}=12$ monomer units to be +4 e. To have an electrically neutral compound, 1600 tosylates were added to the simulation box. The MD simulations start with water equilibration within three steps. First, an energy minimization is performed with steepest descent algorithm. Then, the equilibration continues with an NPT (Constant Number of particles, Pressure, and Temperature) ensemble for 200 ns with a 20 fs time step with position restraints to both the PEDOT and tosylate molecules. When the water is equilibrated, we perform an NPT simulation with the semiisotropic ${ }^{60}$ at 1 bar for $200 \mathrm{~ns}$ with a 20 fs time step. The simulation temperature was set to 300 $\mathrm{K}$ during the equilibrations using the Berendsen thermostat. ${ }^{60}$ With a PEDOT:Tos mixture equilibrated in solution, we process to dry out the system ${ }^{56,61}$ For the evaporation process, we gradually remove water molecules in 100 steps and at last, we study the system with no water inside. At each evaporation step, randomly chosen water molecules were removed and the 
above MD equilibration procedure was repeated for each step. The representative snapshots of the system upon the evaporation process is depicted in Figure 2a. The thickness of the resulting dry film is about $4 \mathrm{~nm}$ which is rather thin compared to the typical experimentally fabricated films ( $\sim 50-200 \mathrm{~nm}$, see Fig. S2). In order to reach a size of the film closer to experimental values, we stack eight $4 \mathrm{~nm}$ films on top of each other in the z-direction to obtain the final thick film.

\section{II.B.b. The simulation box for the cyclic voltammetry}

Having the thick PEDOT:Tos film ready, we place it in a larger box on top of a graphite substrate and add water on top of the polymer film as shown in Figure $2 b$. The substrate used in our simulations is based on a MARTINI model for graphite as used in reference ${ }^{62}$. We have used the substrate of five layers of graphite sheets, frozen at the bottom of the simulation box. Here, it should be noted that the main reason for the presence of the substrate, is to mimic the condition of the EQCM experiment and to avoid water penetration into the film due to the periodic boundary conditions. (Note that the effect of substrate on the morphology and electrical mobility of PEDOT:Tos has been comprehensively computationally investigated in ref ${ }^{63}$ ).

Having the final system prepared, we start to investigate the changes in morphology of PEDOT:Tos film upon cyclic voltammetry, starting with a fully oxidized PEDOT at 33\% oxidation level. The initial box dimensions are $20 \times 20 \times 52 \mathrm{~nm}$ in $x, y$, and $z$ directions, respectively. The MD simulations for the whole cyclic voltammetry procedure is as follows: first an energy minimization is run followed by a short NPT for 5 ns with 1 fs time step. Then a production run is performed for $100 \mathrm{~ns}$ with 5 fs timestep. Semi-isotropic pressure of 1 bar is applied separately in $x y$ plane and $z$ direction using Berendsen barostat. During all the simulation process, the temperature was kept at $\mathrm{T}=400 \mathrm{~K}$ using the modified Berendsen 
thermostat (V-rescale). ${ }^{64}$ The method used for the long-range Coulomb electrostatic interactions is Particle Mesh Ewald ${ }^{65}$ for all simulations with the cutoff of $1.2 \mathrm{~nm}$. Visualization has been made with the VIA-MD software. ${ }^{66}$

\section{II.B.c. Computational cyclic voltammetry}

In order to study the changes in morphology of PEDOT:Tos film upon cyclic voltammetry (CV), we need to mimic the CV condition computationally. To do so, we start with changing the oxidation level on PEDOT chains and at each step a corresponding number of counterions is added to the box in order to preserve the electroneutrality of the system. Starting with fully oxidized PEDOT at 33\%, the oxidation level on PEDOT is reduced to $0 \%$ in 4 steps: $33 \%$, $25 \%, 16 \%, 8 \%, 0 \%$, as the procedure is shown in Figure 2c. During the first reduction cycle, a considerable amount of tosylates is released from the film into the electrolyte. In the condition of experimental $\mathrm{CV}$, these tosylates are washed out away from the film into reservoirs due to the flow of the electrolyte and therefore, they cannot re-enter the film in subsequent cycles. Thus, after each full reduction we remove the tosylates diffused into the electrolyte as shown in the middle panel in Figure 2c. During the oxidation (which is also done in the same 4 steps but in the reversed order) the charge on PEDOT is increased from $0 \%$ to $33 \%$, and at each oxidation step the corresponding number of chlorines is added to the electrolyte, while the number of sodium ions was kept the same. During the reduction, chlorines are removed in each step until the polymer film is fully reduced. Here, the diffused tosylates in the electrolyte are removed and during the consecutive oxidation process, the chlorines are added. The whole mechanism for the computational cyclic voltammetry is depicted in Figure 2c.

In the present study, we calculate the Solvent Accessible Surface Area (SASA) ${ }^{67}$ as implemented in the GROMACS software. The SASA, calculated based on the double cubic 
lattice method, is defined as the area swept by the center of a sphere probe with the radius of a coarse-grained water molecule $(4 \AA)$ rolling around the film consisting of PEDOT chains, tosylate counterions, sodium ions and chlorines. In the present work, we also provide information on how the film mass changes upon CV. For that, we define a boundary for the film where the density of PEDOT and water becomes equal. Having the film boundary defined, we study the changes in the film mass and the film composition.

(a)
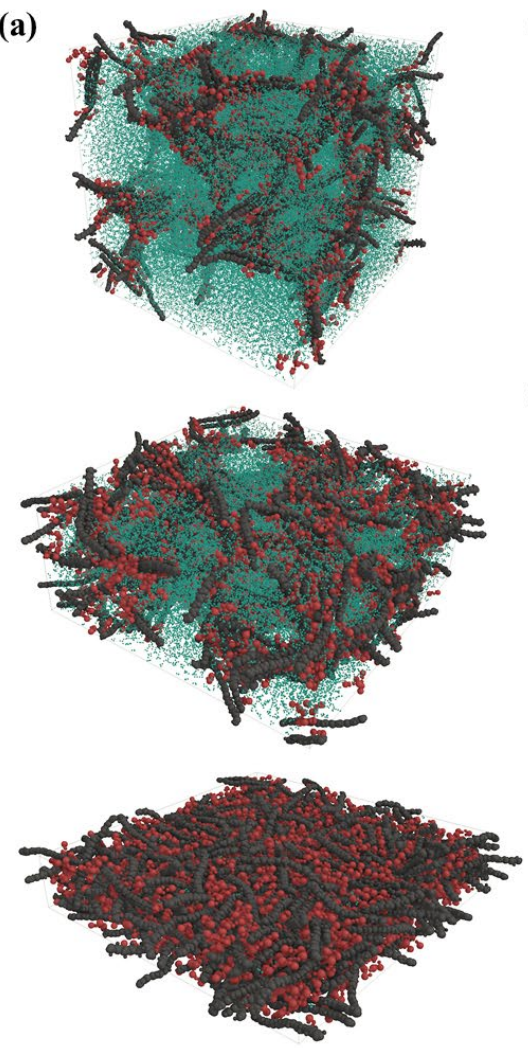

(b)

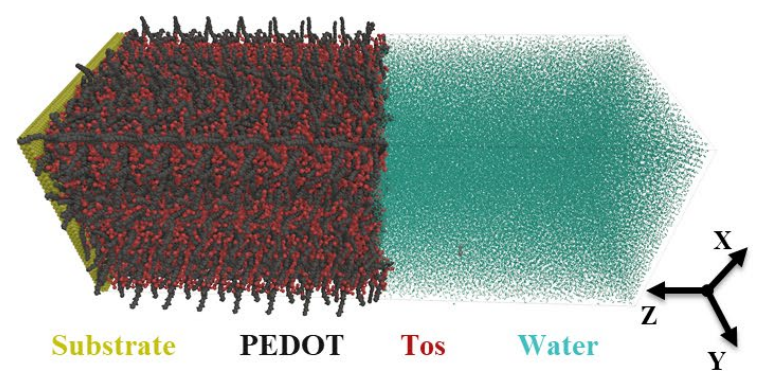

(c)

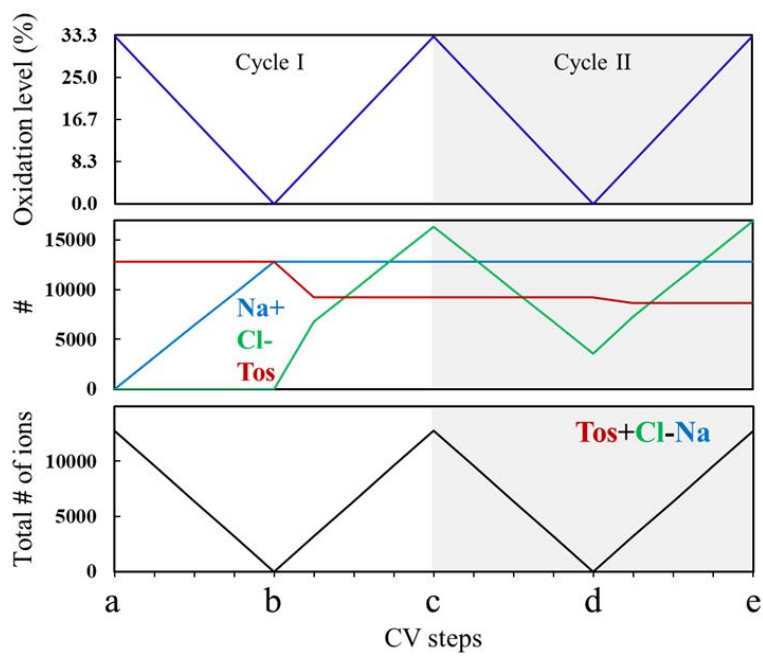

Figure 2. Description of the system under study. (a) evaporation process and formation of a dry film. (b) simulation box containing PEDOT:Tos film in electrolyte. (c) mechanism for the computational cyclic voltammetry.

\section{Results and Discussions}




\section{III.A. Changes in the morphology of the PEDOT:Tos film in MD simulations upon cyclic voltammetry}

This section focuses on Molecular Dynamic simulations investigating changes in the morphology including water intake, swelling and exchange of counterions in PEDOT:Tos film during CV. Figure 3a-e shows the snapshots of the system and the corresponding density profiles throughout the redox process during two first cycles, starting from (a) the fully oxidized state $\left(\mathrm{C}_{\mathrm{ox}}=33 \%\right)$, to (b) the first fully reduced state $\left(\mathrm{C}_{\mathrm{ox}}=0 \%\right)$, (c) the second fully oxidized state $\left(\mathrm{C}_{\mathrm{ox}}=33 \%\right),(\mathrm{d})$ the second fully reduced state $\left(\mathrm{C}_{\mathrm{ox}}=0 \%\right)$, and finally (e) the fully oxidized state $\left(\mathrm{C}_{\mathrm{ox}}=33 \%\right)$ at the end of the second cycle. The labels a, b, c, d, and e in Figure 2c, depicts the corresponding oxidation levels shown in Figure 3.

Let us first describe the changes in the morphology during the first cycle, Figure 3a-c. At the beginning of the simulations, starting with the system at 33\% oxidation level (Figure 3a), there is no water uptake by the film. After reducing the polymer film gradually to $0 \%$, there is a significant amount of tosylates leaving the film toward the electrolyte as shown in Figure $3 \mathrm{~b}$. In particular, during reduction holes are extracted and then some tosylates are released from the polymer matrix while other remain in the film. For the fully reduced film (Fig. 3b) the tosylates that remained within the film are compensated by $\mathrm{Na}+$ that entered the film. The $\mathrm{Na}+$ ions entering the film are surrounded by the water molecules in the hydration shells. These water molecules follow the ions and therefore the water is up taken by the film upon the first reduction process. Having obtained the fully reduced system, we proceed with the cyclic voltammetry and oxidize the film in a step-by-step manner. As described in the method section, to mimic the experimental $\mathrm{CV}$ conditions, we added chlorines in the electrolyte upon the oxidation process. Also, after each full reduction we remove the tosylates diffused into the bulk electrolyte. Figure $3 \mathrm{c}$ shows the fully oxidized state at the end of the first cycle. As shown, sodium ions are released 
from the film and return back to the electrolyte. Release of the sodium ions correlates with the insertion of tosylates and chlorines during the oxidation process.

Starting the second cycle, we begin with the oxidized system and perform the reduction procedure. Figure 3d shows the second full reduction where tosylates and chlorines are released out of the film to the electrolyte and sodium ions are injected into the polymer matrix. After this we continue our computational $\mathrm{CV}$ with oxidizing the polymer again to $33 \%$. As shown in Figure $3 \mathrm{e}$, during oxidation the sodium and chlorines ions are expelled from and injected into the film, respectively. The step-by-step density profiles are shown in Fig. S3.

a) Cycle I, $\mathrm{C}_{\mathrm{ox}}=33 \%$
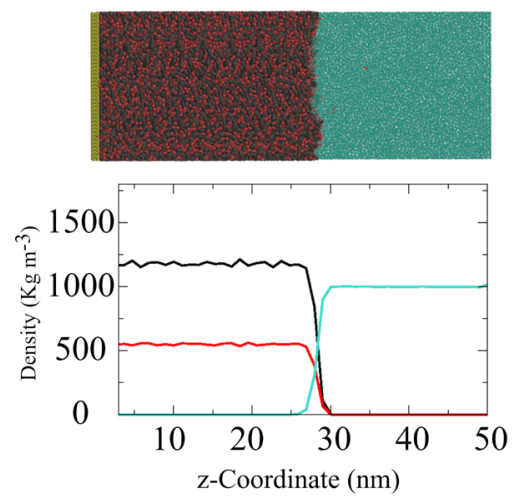

d) Cycle II, $\mathrm{C}_{\mathrm{ox}}=0 \%$
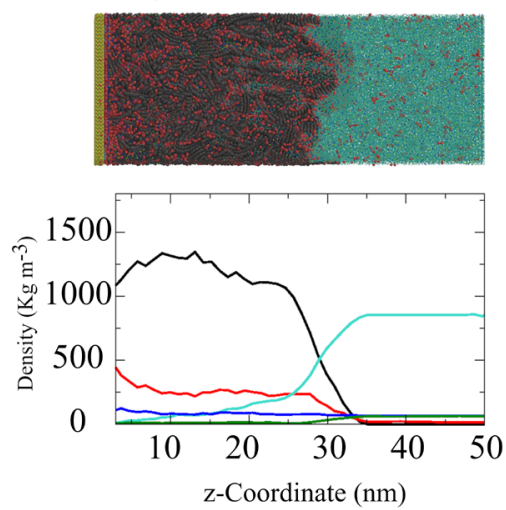

PEDOT TOS b) Cycle I, $\mathrm{C}_{\mathrm{ox}}=0 \%$
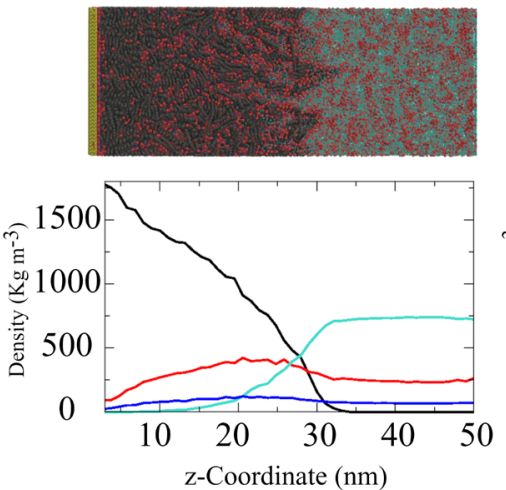

e) Cycle II, $\mathrm{C}_{\mathrm{ox}}=33 \%$
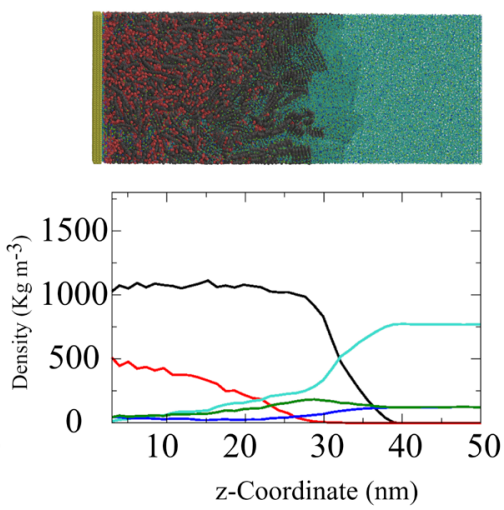

Water $\mathbf{N a}+\mathbf{C l}-$ c) Cycle I, $\mathrm{C}_{\mathrm{ox}}=33 \%$

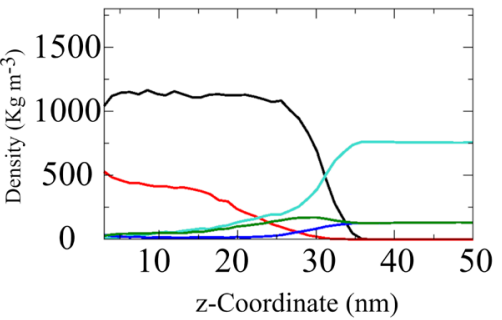


Figure 3. Density profiles and the corresponding snapshots of the system under study during cyclic voltammetry. (labels a, b, c, d, and e in Figure 2c, depicts the corresponding oxidation levels shown here) (a) Beginning of the simulations with the system at 33\% oxidation level. (b) The first full reduction at $0 \%$ oxidation level. (c) End of the first cycle with the system at 33\% oxidation level. (d) Second full reduction at $0 \%$ oxidation level. (e) End of the second cycle with the system at $33 \%$ oxidation level.

To investigate the changes in the morphology of the film we calculate morphological parameters such as volume and solvent accessible surface area (SASA) of the film upon redox processes. The total volume of the film increases during the CV (Figure 4a). During the first reduction, the volume of the film is nearly constant while the most significant increase occurs after the first reduction. There is a trend of increase of the volume corresponding to the expansion of PEDOT:Tos film over the simulated cycles.

The solvent accessible surface area (SASA) is a good measure on how much water is uptaken by the film and how the film evolves during the redox process. Figure $4 \mathrm{~b}$ and Fig. S4 show the calculated SASA of the film including PEDOT chains, tosylate counterions, sodium ions and chlorines present in the system upon the cyclic voltammetry. The SASA shows a sharp increase during the first reduction where sodium ions penetrate inside the film and bring water with them. After the first reduction, the system goes through the oxidation process where sodium ions are released from the film accompanied by the insertion of chlorines into the film. Therefore, SASA stays nearly constant afterwards. However, in the second cycle, SASA starts to increase again. This can be explained by the movement of tosylates. During reduction SASA increases which coincides with the release of tosylates toward the electrolyte, such that the accessible area for the solvent increases inside the film when tosylates leave. Whereas during oxidation, tosylates cease to leave the film and therefore SASA does not increase anymore. The solvent accessible surface area reaches a plateau after two cycles.

The total mass of the film decreases during reduction and increases during oxidation (Figure 4c). This is due to ions/counterions exchange between the film and the electrolyte. The decrease 
of the film mass upon reduction is due to the release of tosylates from the polymer matrix (see Figure 4d). Release of Tos is accompanied by the insertion of sodium ions into the film. Since tosylates leaving the film are much heavier than the sodium ions entering the film, the total mass of the film decreases. During the oxidation process, the total mass increases due to the insertion of chlorines together with water molecules and release of the sodium ions from the polymer matrix. However, when the film is oxidized again, the film mass continues to decrease at the first steps and then it increases. Specifically, MD simulations revealed that the initial decrease of the mass during early stages of the oxidation process is because of $\mathrm{Na}+$ ions expulsion from the film where the ions take a number of water molecules with them. Then the mass starts to increase due to the injection of $\mathrm{Cl}$ - ions surrounded by the hydration shells.

It is noteworthy that the polymer film exhibits a general trend of losing mass during cyclic voltammetry. This is due to the release of the heavy tosylate counterions from the film to the bulk electrolyte. Thus, each time that the system is back to its initial oxidation level (33\%), it has lost a significant amount of tosylates, which is replaced by chlorine ions. This is consistent with previous work by Rudd et all ${ }^{32}$ on PEDOT:TOS films where they verified with XPS the partial replacement of tosylates by various anions during electrochemical cycling. Hence, the total mass of the film is lower than its initial value which is about $88 \%$ of the initial mass after the two cycles in our calculations. Also, during the second cycle, the mass change between the most oxidized and most reduced states is $10 \%$. It should be also noticed that the surface roughness of the film is increased during the cyclic voltammetry, which is due to the disturbance caused by the ionic motion through the polymer-electrolyte interface.

A change in the number of species inside the film during $\mathrm{CV}$ is presented in Figure 4d. As expected, the number of PEDOT chains in the film does not change. The number of tosylates inside the film decreases upon reduction and, as expected, is almost constant during the oxidation process where tosylates tend to stay inside the film and do not leave the polymer 
matrix anymore. After the first and second cycles $40 \%$ and $10 \%$ of the tosylates are lost, respectively. Sodium ions are injected into the film during reduction and leave the film during oxidation, therefore that their number increases and decreases, respectively. However, chlorines have the opposite trend since they are injected to the film upon oxidation and are released during the reduction process. The number of water molecules inside the film increases because ions injected into the polymer matrix bring water molecules with them (see Fig. S5). It is expected that after several cycles the volume expansion diminishes because there is practically no space in the film left to accommodate water molecules. Based on our calculations, during reduction each sodium ion brings $\sim 4$ water molecules inside the film and during oxidation each chlorine brings $\sim 4$ water molecules inside the film, as well. This result is similar to the one reported for PEDOT:PSS. 

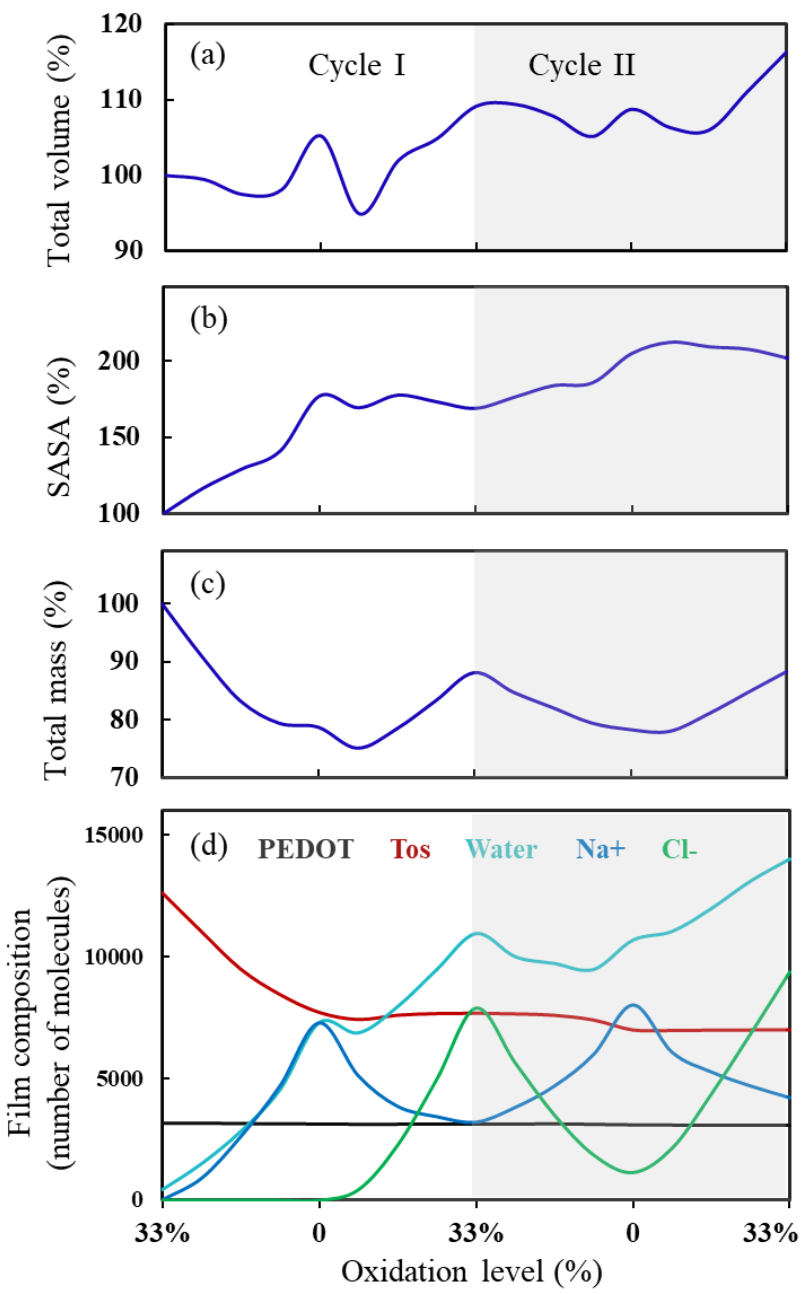

Figure 4. Changes in the morphology of the film upon cyclic voltammetry. (a) Total volume of PEDOT. (b) Solvent accessible surface area (SASA) for PEDOT. (c) changes in the total mass of the film and (d) film composition (number of molecules).

III.B. e-QCMD studies of PEDOT:Tos films and interpretation of the experimental results

The mass change of PEDOT:Tos films during electrochemical cycling was monitored experimentally by electrochemical quartz crystal microbalance. PEDOT:Tos films were prepared via oxidative polymerization on Si substrates as described in detail in the methods. 
The films were subsequently transferred onto Au coated quartz crystals and were mounted onto the e-QCMD electrochemical cell as the working electrode. Initially required baselines in air and electrolyte as well as transition phases were recorded (Fig. S6). With the baselines, the dry thickness of the films in dry state was assessed (Fig. S2). In the cyclic voltammetry experiments, we then, we electrochemically reduced and oxidized the films by cyclic voltammetry between $(+0.5,-0.7 \mathrm{~V})$ with $1 \mathrm{mV} / \mathrm{s}$ scan rate (Fig. S7, S8) while at the same time we monitored the mass changes via the frequency change of the QCM crystal. In each electrochemical cycle at $0.5 \mathrm{~V}$ and $-0.7 \mathrm{~V}$ the potential was kept constant for 30 minutes in order to ensure that the films are completely oxidized or reduced. The PEDOT:Tos film as prepared is in the oxidized state. On the onset of the experiment by application of $0.5 \mathrm{~V}$ we observed an immediate mass change (within $1 \mathrm{sec}$ ) of about $1.7 \%$ indicating that the film is oxidized further from its pristine state (Fig. 5 and Fig. S9) with the inclusion of Cl- dopant ions. For thicker films the initial mass change is even larger, up to about $13.9 \%$ from the pristine state, see Fig. S10. This conclusion is corroborated by UV-VIS-NIR spectroscopy that shows that when PEDOT:TOS film is electrochemically oxidized by applying $+0.5 \mathrm{~V}$ only for few seconds it goes to higher oxidation state than the pristine PEDOT:TOS film. During reduction $(+0.5 \mathrm{~V}$ to $-0.7 \mathrm{~V})$ the mass decreases with potential in a monotonic fashion, while during oxidation $(-0.7$ to $+0.5 \mathrm{~V})$ the mass initially decreases with applied potential, then increases with a small rate and then increases with a larger rate (Fig. 5b and Fig. S8). The decrease of mass during reduction can be explained by the expulsion of dopant ions from the films while the increase of mass during oxidation by inclusion of dopant ions in the film. The fact that we observe an initial decrease of the mass during oxidation can be explained by expulsion of $\mathrm{Na}+$ ions that have entered the film during reduction as is also shown in the findings of the MD simulations. Therefore, during reduction although the mass overall decreases with the expulsion of the dopant ions, $\mathrm{Na}+$ ions also enter the film to neutralize dopants. In subsequent cycles similar trends are observed with a mass 
change of 10 to $14 \%$ between the fully reduced to the fully oxidized state. These observations are in excellent agreement with the MD simulations where the relative mass change between the most oxidized and reduced states is $\sim 10 \%$.

Note that experimental rates of the mass change cannot be directly compared with the simulated results, because in the simulations we assumed a linear change of the oxidation level which takes place homogeneously over the entire film. This is a simplified model because in experimental samples the temporal and spatial propagation of the reduction/oxidation is not expected to be linear and homogeneous and is a result of a complex interplay between the electron and ion motion.

a)

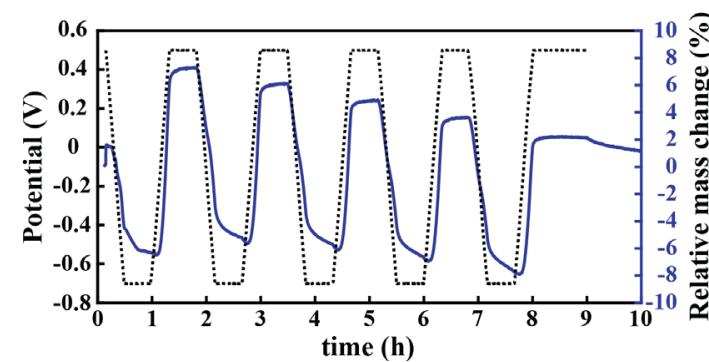

b)

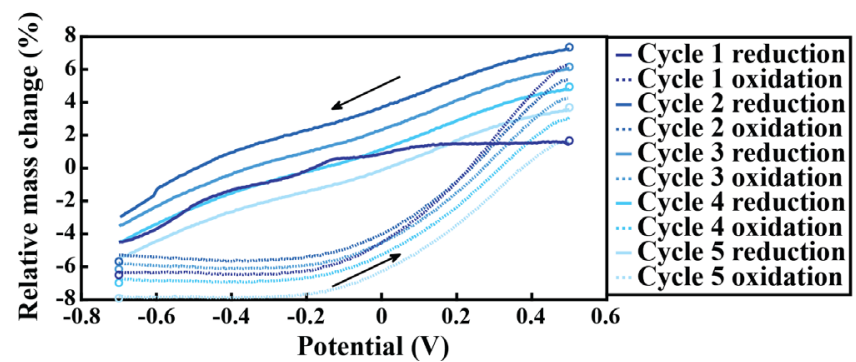

Figure 5. e-QCMD measurements. a) PEDOT:Tos mass change in respect to the pristine state ( $\sim 65 \mathrm{~nm}$ dry thickness) over time during electrochemical cycling. The applied potential change over time is shown in dotted line (The frequency and dissipation changes can be found in Figure S11). b) Relative mass of PEDOT:Tos vs applied potential as shown in (a). The circles indicate the relative mass change at the end of the potentiostatic phase.

Furthermore, it must be noted that during the potential sweeps, we observed a strong oxygen reduction reaction (ORR) even though the samples and the electrolyte were purged with nitrogen (Fig. S7.). In order to ensure that the ORR was not impacting the recorded mass changes we performed experiments at different scan rates $(1,10,50 \mathrm{mV} / \mathrm{s})$. We found that we always observe the same mass change irrespectively of the scan rate and the magnitude of the ORR (Figure S12). This is in line with previous observations for PEDOT with other dopants. ${ }^{41}$ 
During the potentiostatic phase of the cycle we observe that the mass change reaches a plateau when the oxidative potential is applied while it keeps decreasing by around $2.5 \%$ in 30 minutes when a reducing potential is applied (Fig. S13). This can be explained by movement of ions with different mobility. Indeed, it is expected that initially the more mobile Cl- ions are expelled while at later time the less mobile tosylates leave the film.

Over the course of the 5 observed cycles, the magnitude of the mass changes abates and the total film mass decreases indicating a gradual loss of mass from the films into the bulk electrolyte, see Fig. 5. We attribute the overall mass change to dopant exchange and not loss of PEDOT, because PEDOT is insoluble in water, which makes it unlikely for individual chains to solubilize. Furthermore, we are cycling the film in a safe electrochemical window and no film degradation was observed after the e-QCMD measurements. In figure S7 a, it is visible in the CVs that the capacitive contribution in the oxidative regime of the $3 \mathrm{rd}$ to 5 th cycle is stable. Hence, as the capacitive effects are linked to the polymer volume, this indicates that there are no major losses of PEDOT occurs. Not least also the MD shows no loss of PEDOT to the electrolyte (Fig. 4d.). The observed overall mass loss again is in line with the results of the MD simulations (Fig. 4 c) that predict the decrease of the mass of the film due to dopant exchange. Owing to the heavier and bulkier nature of tosylate in comparison to $\mathrm{Cl}-$, Tosylate is gradually replaced in the film by $\mathrm{Cl}^{-}$, thus causing the loss of the film mass. The QCM though, records overall mass change and cannot give information on the type of ions that enter or leave the film therefore in order to getter better inside on the ionic composition of the films we performed XPS that will be discussed in the next section.

\section{III.C. XPS studies of the polymer films}


To investigate further the ions that are participating in the electrochemical reduction and oxidation of PEDOT:TOS we performed XPS. By examining the variation of S $2 p$ spectral features (see figure S14) we could define the relative change of the content of Tos in the reduced and oxidized PEDOT:TOS film. The films were prepared as for the QCM experiment but on FTO substrates and the same electrochemical procedure (See Figure S15 b) as in e-QCM experiments was applied. As XPS is performed in vacuum, after electrochemically reducing and oxidizing the films, they were removed from the electrolyte and dried gently with N2. In order to ensure that the films retained the reduced and oxidized state when dried, before XPS characterization, we performed UV-VIS-NIR absorption spectroscopy. We observed that the reduced film shows the characteristic neutral band of PEDOT at $600 \mathrm{~nm}$ but also still absorption at NIR region (peaks at $\approx 900 \mathrm{~nm}$ and $\geq 1500 \mathrm{~nm}$ ) indicating that the film is not fully reduced. (A detailed analysis of the origin of the peaks in the absorption spectra of thiophene-based polymers and their evolution with the doping level can be found in ref. ${ }^{68}$ ). This can be explained by the partial oxidation of the film when the reducing potential is not applied any more, but the film is still connected in the electrochemical cell. Furthermore, the exposure of the film in oxygen when is removed from the electrochemical cell can induce oxidation, as well.

XPS data in Table 1, present the atomic ratio of the studied PEDOT:Tos film. Here we set the S content of the monomer ( $2 \mathrm{~S}$ in EDOT) $100 \%$, as we consider PEDOT will always be kept inside the film. The S content of Tos (one $\mathrm{S}$ in Tos) is about $32.8 \%$ in the pristine film, indicating that $\mathrm{S}$ ratio between EDOT and Tos is about 100:32.8 $(\sim 3: 1)$. The same analogy works for the reduced and oxidized states, as well. Based on the XPS measurements, the pristine film shows that the atomic ratio of Tos to PEDOT is about $32.4 \%$ after deconvolution of the $\mathrm{S}$ $2 \mathrm{p}$ peak (figure S14 a and Table 1). After the reduction process, the atomic ratio is decreased to $8.6 \%$ (figure $\mathrm{S} 14 \mathrm{~b}$ and Table 1). In addition, there is $8.8 \% \mathrm{Cl}^{-}$and $5.8 \% \mathrm{Na}^{+}$present in the system. Therefore, the total oxidation level is about $11.6 \%$. This finding is in agreement with 
the partial oxidation of the reduced film as observed in the UV-VIS -NIR as mentioned above. The presence of $\mathrm{Cl}^{-}$also supports the fact that the film has been re-oxidized before it was removed from the electrochemical cell.

In the oxidated state, the atomic ratio between Tos to PEDOT becomes 12\% (figure S14 c and Table 1). The amount of $\mathrm{Cl}^{-}$increases to about $22.6 \%$ while the amount of $\mathrm{Na}+$ decreases to $3.9 \%$. From these findings one can derive, that as a result, the final oxidation level is about $30.7 \%$, very close to that of the pristine PEDOT:Tos film, which is in line with the UV-VISNIR spectra. The XPS study confirms the MD simulation and e-QCM findings that in the oxidation of the film Tos was replaced by the more mobile $\mathrm{Cl}^{-}$ions.

Table 1. Atomic ratio between EDOT, Tos, $\mathrm{Cl}$, and $\mathrm{Na}$ in the film at different states in XPS measurements vs. MD simulations (a,b,c in the MD section correspond to the state of the system as shown in Figure 2c)

\begin{tabular}{|c|c|c|c|c|c|c|}
\hline & & $\begin{array}{c}\text { \% EDOT } \\
\text { (S) }\end{array}$ & \% Tos (S) & \% Cl & \% Na & $\begin{array}{c}\text { \% Oxidation } \\
\text { level }\end{array}$ \\
\hline \multirow{2}{*}{$\tilde{\Sigma}$} & Pristine & 100 & 32.4 & 0 & 0 & 32.4 \\
\cline { 2 - 7 } & Reduced & 100 & 8.6 & 8.8 & 5.8 & 11.6 \\
\cline { 2 - 7 } & Oxidized & 100 & 12 & 22.6 & 3.9 & 30.7 \\
\hline \multirow{2}{*}{$\Sigma$} & Pristine (a) & 100 & 33.3 & 0 & 0 & 33.3 \\
\cline { 2 - 7 }$\Sigma$ & Reduced (b) & 100 & 20 & 0 & 20 & 0 \\
\cline { 2 - 7 } & Oxidized (c) & 100 & 20.7 & 21.1 & 8.5 & 33.3 \\
\hline
\end{tabular}

UV-VIS-NIR spectroscopy revealed that the reduced system shows a clear deviation from the system in the oxidized state (figure S15). MD calculations shed light on the nature of this deviation, that is, exchange of ions/counter-ions and in particular, release of Tos from the film during reduction process (Figure 4d). XPS analysis confirmed that after the electroreduction of the polymer, a portion of Tos leave the polymer which is consistent with MD simulations (see 
Figure S14 and Table 1). Also, both XPS and MD show that when the system is re-oxidized, a large portion of Tos is replaced by ions that entered the film during the redox process. However, there are some discrepancies between the experiment and simulations. That is, in the simulations the system is reduced completely to $0 \%$ while the reduced experiment sample has still an oxidation level about $11.6 \%$. This incomplete reduction may come from the fact that the film re-oxidized before it was removed from the electrochemical cell. This is further supported by the presence of $\mathrm{Cl}$ ions in the reduced film. There is also a difference in the chemical composition of the reduced state where XPS shows a portion of $\mathrm{Cl}^{-}$content in the system while there is no $\mathrm{Cl}^{-}$in $\mathrm{MD}$ (see Table 1). This comes back to the strategy we chose to perform computational cyclic voltammetry (see section II.B.c. and Figure 2c ). That is, during reduction $\mathrm{Na}^{+}$is added to keep the simulation box charge-neutral whereas $\mathrm{Cl}$ - is added during oxidation. Thus, there is no $\mathrm{Cl}^{-}$defined in the first reduction state in $\mathrm{MD}$ to appear here. Also the fact that $\mathrm{Cl}^{-}$is observed in the experimental reduced state can be explained by the partial oxidation of the reduced film. That is if the sample was completely reduced, there should have not been any Cl- detected as it is predicted by MD simulations.

\section{Conclusions}

We studied the ion exchange and swelling of PEDOT:Tos films upon cyclic voltammetry both experimentally by electrochemical quartz crystal microbalance with dissipation and computationally by coarse grained molecular dynamics simulations. By combining the experimental measurements such as e-QCM, UV-VIS-NIR absorption spectroscopy, and XPS with "computational microscopy", we were in a unique position to provide a detailed atomistic understanding of the water intake and ion injection during cyclic voltammetry. We 
showed that the film underwent significant changes in morphology and mass during the redox processes. We observed that the film lost its mass during reduction as Tosylate and $\mathrm{Na}$ was expelled and gained mass during oxidation mainly due to the uptake of anions, i.e. Tosylate and

Cl. Both e-QCM experiments and simulations showed that a relative mass change between the most oxidized and reduced states was around 10\%-14\%. With increasing cycle number the total film mass gradually decreased because Tosylate in the film was replaced by more agile $\mathrm{Cl}$ anions during cycling. XPS measurements and MD simulations agreed on the fact that a portion of Tos is replaced by $\mathrm{Cl}$ - during the voltammetry cycles. In addition, MD simulations showed that during redox process the volume of the film was gradually increased. Based on our calculations we concluded that each ion brought $\sim 4$ water molecules into the film. We believe that our study combing the experimental investigation with computational insight provided important understanding to the underlying molecular processes of the PEDOT:Tos film during cyclic voltammetry that has not been available previously.

\section{Author Information}

Corresponding Author

Igor Zozoulenko - Laboratory of Organic Electronics, ITN, Linköping University, 60174 Norrköping, Sweden;

orcid.org/0000-0002-6078-3006;

Email: igor.zozoulenko@liu.se

Authors

Najmeh Delavari - Laboratory of Organic Electronics, ITN, Linköping University, 60174 Norrköping, Sweden; 
Johannes Gladisch - Laboratory of Organic Electronics, ITN, Linköping University, 60174 Norrköping, Sweden;

Ioannis Petsagkourakis - Laboratory of Organic Electronics, ITN, Linköping University, 60174 Norrköping, Sweden;

Xianjie Liu - Laboratory of Organic Electronics, ITN, Linköping University, 60174 Norrköping, Sweden;

Mohsen Modarresi - Department of Physics, Ferdowsi University of Mashhad, Mashhad, Iran;

Mats FahIman - Laboratory of Organic Electronics, ITN, Linköping University, 60174 Norrköping, Sweden;

Eleni Stavrinidou - Laboratory of Organic Electronics, ITN, Linköping University, 60174 Norrköping, Sweden;

Mathieu Linares - Laboratory of Organic Electronics, ITN, Linköping University, 60174 Norrköping, Sweden; Group of Scientific Visualization, Department of Science and Technology (ITN), Campus Norrköping, Linköping University, SE-60174 Norrköping, Sweden; Swedish e-Science Center (SeRC), Linköping University, SE-581 83 Linköping, Sweden

\section{Acknowledgements}

This work was supported by the Swedish Research Council (projects 2016-05990 and 201704474). IZ thank the Swedish Government Strategic Research Area in Materials Science on 
Advanced Functional Materials at Linköping University (Faculty Grant SFO-Mat-LiU No. 2009-00971) for support. ML thanks the Swedish e-Science Research Centre (SeRC) for financial support. The computations were performed on resources provided by the Swedish National Infrastructure for Computing (SNIC) at NSC and HPC2N.

\section{Supporting information}

Step-by-step density profiles during cyclic voltammetry. Snapshots representing the evolution of the system under study during cyclic voltammetry. Changes in solvent accessible surface area and volume of the film during the cyclic voltammetry. Frequency change of a PEDOT:Tos film following the change from air to electrolyte $(0.01 \mathrm{M} \mathrm{NaCl})$. Rapid relative mass change of a pristine PEDOT:Tos film upon application of oxidative potential. Absorption spectroscopy of PEDOT:Tos films in different oxidation states. Frequency change of three similarly prepared films and the corresponding relative mass changes of one film. Current versus voltage during the potential sweeps' phases corresponding to the mass changes. Detailed view of the relative mass changes between $-0.7 \mathrm{~V}$ and $0 \mathrm{~V}$ in the oxidative potential sweep. Detailed view of the relative mass changes during potentiostatic phases of the switching cycles at $0.5 \mathrm{~V}$ (oxidation) and $-0.7 \mathrm{~V}$ (reduction). Relative mass change (in respect to the initial mass) during reduction and oxidation in consecutive electrochemical cycles. Investigations on the effect of the oxygen reduction reaction on the frequency changes. The deconvolution of XPS S2p peaks for the pristine, reduced, and oxidized PEDOT:Tos.

\section{References}

1. Groenendaal, L.; Zotti, G.; Aubert, P. H.; Waybright, S. M.; Reynolds, J. R. Electrochemistry of poly (3, 4-alkylenedioxythiophene) derivatives. Advanced Materials 2003, 15 (11), 855-879. 

J.; Yang. J. The Morphology of Poly (3, 4-Ethylenedioxythiophene). Polym. Rev 2010, 50, 340-384.

3. Elschner, A.; Kirchmeyer, S.; Lovenich, W.; Merker, U.; Reuter, K., PEDOT: principles and applications of an intrinsically conductive polymer. CRC Press: 2010.

4. Petsagkourakis, I.; Kim, N.; Tybrandt, K.; Zozoulenko, I.; Crispin, X. Poly (3, 4ethylenedioxythiophene): chemical synthesis, transport properties, and thermoelectric devices. Advanced Electronic Materials 2019, 5 (11), 1800918.

5. $\quad \mathrm{He}, \mathrm{J}$.; Su, J.; Wang, J.; Zhang, L. Synthesis of water-free PEDOT with polyvinylpyrrolidone stabilizer in organic dispersant system. Organic Electronics 2018, 53, 117-126.

6. Winther-Jensen, B.; West, K. Vapor-phase polymerization of 3, 4ethylenedioxythiophene: a route to highly conducting polymer surface layers. Macromolecules 2004, 37 (12), 4538-4543.

7. Khan, Z. U.; Bubnova, O.; Jafari, M. J.; Brooke, R.; Liu, X.; Gabrielsson, R.; Ederth, T.; Evans, D. R.; Andreasen, J. W.; Fahlman, M. Acido-basic control of the thermoelectric properties of poly (3, 4-ethylenedioxythiophene) tosylate (PEDOT-Tos) thin films. Journal of Materials Chemistry C 2015, 3 (40), 10616-10623.

8. $\quad$ Yi, C.; Zhang, L.; Hu, R.; Chuang, S. S.; Zheng, J.; Gong, X. Highly electrically conductive polyethylenedioxythiophene thin films for thermoelectric applications. Journal of Materials Chemistry A 2016, 4 (33), 12730-12738.

9. Wang, H.; Ail, U.; Gabrielsson, R.; Berggren, M.; Crispin, X. Ionic Seebeck effect in conducting polymers. Advanced Energy Materials 2015, 5 (11), 1500044.

10. Brooke, R.; Edberg, J.; landolo, D.; Berggren, M.; Crispin, X.; Engquist, I. Controlling the electrochromic properties of conductive polymers using UV-light. Journal of Materials Chemistry $C$ 2018, 6 (17), 4663-4670.

11. Stavrinidou, E.; Leleux, P.; Rajaona, H.; Khodagholy, D.; Rivnay, J.; Lindau, M.; Sanaur, S.; Malliaras, G. G. Direct measurement of ion mobility in a conducting polymer. Advanced Materials 2013, 25 (32), 4488-4493.

12. Berggren, M.; Crispin, X.; Fabiano, S.; Jonsson, M. P.; Simon, D. T.; Stavrinidou, E.; Tybrandt, K.; Zozoulenko, I. Ion electron-coupled functionality in materials and devices based on conjugated polymers. Advanced Materials 2019, 31 (22), 1805813.

13. Nilsson, D.; Chen, M.; Kugler, T.; Remonen, T.; Armgarth, M.; Berggren, M. Bi-stable and dynamic current modulation in electrochemical organic transistors. Advanced Materials 2002, 14 (1), 51-54.

14. Wan, A. M.; Schur, R. M.; Ober, C. K.; Fischbach, C.; Gourdon, D.; Malliaras, G. G. Electrical control of protein conformation. Advanced materials 2012, 24 (18), 2501-2505.

15. Svennersten, K.; Bolin, M. H.; Jager, E. W.; Berggren, M.; Richter-Dahlfors, A. Electrochemical modulation of epithelia formation using conducting polymers. Biomaterials 2009, 30 (31), 6257-6264.

16. Bongo, M.; Winther-Jensen, O.; Himmelberger, S.; Strakosas, X.; Ramuz, M.; Hama, A.; Stavrinidou, E.; Malliaras, G. G.; Salleo, A.; Winther-Jensen, B. PEDOT: gelatin composites mediate brain endothelial cell adhesion. Journal of Materials Chemistry B 2013, 1 (31), 3860-3867.

17. Iandolo, D.; Ravichandran, A.; Liu, X.; Wen, F.; Chan, J. K.; Berggren, M.; Teoh, S. H.; Simon, D. T. Development and characterization of organic electronic scaffolds for bone tissue engineering. Advanced healthcare materials 2016, 5 (12), 1505-1512.

18. Tehrani, P.; Hennerdal, L.-O.; Dyer, A. L.; Reynolds, J. R.; Berggren, M. Improving the contrast of all-printed electrochromic polymer on paper displays. Journal of Materials Chemistry 2009, 19 (13), 1799-1802.

19. Hong, K.; Kim, S. H.; Yang, C.; An, T. K.; Cha, H.; Park, C.; Park, C. E. Photopatternable, highly conductive and low work function polymer electrodes for high-performance n-type bottom contact organic transistors. Organic Electronics 2011, 12 (3), 516-519. 

ethylenedioxythiophene): tosylate conductive polymer microelectrodes for transmitter detection. Analyst 2012, 137 (8), 1831-1836.

21. Jonsson, A.; Song, Z.; Nilsson, D.; Meyerson, B. A.; Simon, D. T.; Linderoth, B.; Berggren, M. Therapy using implanted organic bioelectronics. Science Advances 2015, 1 (4), e1500039.

22. Malti, A.; Edberg, J.; Granberg, H.; Khan, Z. U.; Andreasen, J. W.; Liu, X.; Zhao, D.; Zhang, H.; Yao, Y.; Brill, J. W. An organic mixed ion-electron conductor for power electronics. Advanced science 2016, 3 (2), 1500305.

23. Cai, M.; Ye, Z.; Xiao, T.; Liu, R.; Chen, Y.; Mayer, R. W.; Biswas, R.; Ho, K. M.; Shinar, R.; Shinar, J. Extremely Efficient Indium-Tin-Oxide-Free Green Phosphorescent Organic Light-Emitting Diodes. Advanced Materials 2012, 24 (31), 4337-4342.

$24 . \quad$ Stutzmann, N.; Friend, R. H.; Sirringhaus, H. Self-aligned, vertical-channel, polymer fieldeffect transistors. Science 2003, 299 (5614), 1881-1884.

25. Kim, D.; Zozoulenko, I. Why Is Pristine PEDOT Oxidized to 33\%? A Density Functional Theory Study of Oxidative Polymerization Mechanism. The Journal of Physical Chemistry B 2019, 123 (24), 5160-5167.

26. Bubnova, O.; Khan, Z. U.; Wang, H.; Braun, S.; Evans, D. R.; Fabretto, M.; Hojati-Talemi, P.; Dagnelund, D.; Arlin, J.-B.; Geerts, Y. H. Semi-metallic polymers. Nature materials 2014, 13 (2), 190. 27. Rivnay, J.; Inal, S.; Collins, B. A.; Sessolo, M.; Stavrinidou, E.; Strakosas, X.; Tassone, C.; Delongchamp, D. M.; Malliaras, G. G. Structural control of mixed ionic and electronic transport in conducting polymers. Nature communications 2016, 7, 11287.

28. Gangopadhyay, R.; Das, B.; Molla, M. R. How does PEDOT combine with PSS? Insights from structural studies. RSC Advances 2014, 4 (83), 43912-43920.

29. Takano, T.; Masunaga, H.; Fujiwara, A.; Okuzaki, H.; Sasaki, T. PEDOT nanocrystal in highly conductive PEDOT: PSS polymer films. Macromolecules 2012, 45 (9), 3859-3865.

30. Wijeratne, K.; Vagin, M.; Brooke, R.; Crispin, X. Poly (3, 4-ethylenedioxythiophene)tosylate (PEDOT-Tos) electrodes in thermogalvanic cells. Journal of Materials Chemistry A 2017, 5 (37), 19619-19625.

31. Muñoz, W. A.; Singh, S. K.; Franco-Gonzalez, J.; Linares, M.; Crispin, X.; Zozoulenko, I. Insulator to semimetallic transition in conducting polymers. Physical Review B 2016, 94 (20), 205202.

32. Rudd, S.; Franco-Gonzalez, J. F.; Kumar Singh, S.; Ullah Khan, Z.; Crispin, X.; Andreasen, J. W.; Zozoulenko, I.; Evans, D. Charge transport and structure in semimetallic polymers. Journal of Polymer Science Part B: Polymer Physics 2018, 56 (1), 97-104.

33. Rivnay, J.; Owens, R. M.; Malliaras, G. G. The rise of organic bioelectronics. Chemistry of Materials 2013, 26 (1), 679-685.

34. Lee, Y. H.; Oh, J.; Lee, S.-S.; Kim, H.; Son, J. G. Highly Ordered Nanoconfinement Effect from Evaporation-Induced Self-Assembly of Block Copolymers on In Situ Polymerized PEDOT: Tos. ACS Macro Letters 2017, 6 (4), 386-392.

35. Aasmundtveit, K.; Samuelsen, E.; Pettersson, L.; Inganäs, O.; Johansson, T.; Feidenhans, R. Structure of thin films of poly (3, 4-ethylenedioxythiophene). Synthetic Metals 1999, 101 (1-3), 561564.

36. Stavrinidou, E.; Winther-Jensen, O.; Shekibi, B. S.; Armel, V.; Rivnay, J.; Ismailova, E.; Sanaur, S.; Malliaras, G. G.; Winther-Jensen, B. Engineering hydrophilic conducting composites with enhanced ion mobility. Physical Chemistry Chemical Physics 2014, 16 (6), 2275-2279.

37. Rolland, N.; Franco-Gonzalez, J. F.; Volpi, R.; Linares, M.; Zozoulenko, I. V. Understanding morphology-mobility dependence in PEDOT: Tos. Physical Review Materials 2018, 2 (4), 045605.

38. Franco-Gonzalez, J. F.; Zozoulenko, I. V. Molecular dynamics study of morphology of doped PEDOT: from solution to dry phase. The Journal of Physical Chemistry B 2017, 121 (16), 42994307.

39. O'sullivan, C.; Guilbault, G. Commercial quartz crystal microbalances-theory and applications. Biosensors and bioelectronics 1999, 14 (8-9), 663-670. 
40.

Wieland, M.; Dingler, C.; Merkle, R.; Maier, J.; Ludwigs, S. Humidity-Controlled Water Uptake and Conductivities in Ion and Electron Mixed Conducting Polythiophene Films. ACS Applied Materials \& Interfaces 2020, 12 (5), 6742-6751.

41. Savva, A.; Wustoni, S.; Inal, S. Ionic-to-electronic coupling efficiency in PEDOT: PSS films operated in aqueous electrolytes. Journal of Materials Chemistry C 2018, 6 (44), 12023-12030.

42. Modarresi, M.; Mehandzhiyski, A.; Fahlman, M.; Tybrandt, K.; Zozoulenko, I. Microscopic Understanding of the Granular Structure and the Swelling of PEDOT: PSS. Macromolecules 2020, 53 (15), 6267-6278.

43. Bischak, C. G.; Flagg, L. Q.; Yan, K.; Rehman, T.; Davies, D. W.; Quezada, R. J.; Onorato, J. W.; Luscombe, C. K.; Diao, Y.; Li, C.-Z. A Reversible Structural Phase Transition by ElectrochemicallyDriven Ion Injection into a Conjugated Polymer. Journal of the American Chemical Society 2020, 142 (16), 7434-7442.

44. Savva, A.; Hallani, R.; Cendra, C.; Surgailis, J.; Hidalgo, T. C.; Wustoni, S.; Sheelamanthula, R.; Chen, X.; Kirkus, M.; Giovannitti, A. Balancing lonic and Electronic Conduction for High-Performance Organic Electrochemical Transistors. Advanced Functional Materials 2020, 30 (11), 1907657.

45. Gladisch, J.; Stavrinidou, E.; Ghosh, S.; Giovannitti, A.; Moser, M.; Zozoulenko, I.; McCulloch, I.; Berggren, M. Reversible Electronic Solid-Gel Switching of a Conjugated Polymer. Advanced Science 2020, 7 (2), 1901144.

46. Petsagkourakis, I.; Pavlopoulou, E.; Cloutet, E.; Chen, Y. F.; Liu, X.; Fahlman, M.; Berggren, M.; Crispin, X.; Dilhaire, S.; Fleury, G. Correlating the Seebeck coefficient of thermoelectric polymer thin films to their charge transport mechanism. Organic Electronics 2018, 52, 335-341.

47. Höök, F.; Rodahl, M.; Brzezinski, P.; Kasemo, B. Energy dissipation kinetics for protein and antibody- antigen adsorption under shear oscillation on a quartz crystal microbalance. Langmuir 1998, 14 (4), 729-734.

48. Savva, A.; Cendra, C.; Giugni, A.; Torre, B.; Surgailis, J.; Ohayon, D.; Giovannitti, A.; McCulloch, I.; Di Fabrizio, E.; Salleo, A. Influence of water on the performance of organic electrochemical transistors. Chemistry of Materials 2019, 31 (3), 927-937.

49. Moser, M.; Hidalgo, T. C.; Surgailis, J.; Gladisch, J.; Ghosh, S.; Sheelamanthula, R.; Thiburce, Q.; Giovannitti, A.; Salleo, A.; Gasparini, N. Side Chain Redistribution as a Strategy to Boost Organic Electrochemical Transistor Performance and Stability. Advanced Materials 2020, 2002748.

50. Marrink, S. J.; Risselada, H. J.; Yefimov, S.; Tieleman, D. P.; De Vries, A. H. The MARTINI force field: coarse grained model for biomolecular simulations. The journal of physical chemistry $B$ 2007, 111 (27), 7812-7824.

51. Berendsen, H. J.; van der Spoel, D.; van Drunen, R. GROMACS: a message-passing parallel molecular dynamics implementation. Computer physics communications 1995, 91 (1-3), 43-56. 52. Van Der Spoel, D.; Lindahl, E.; Hess, B.; Groenhof, G.; Mark, A. E.; Berendsen, H. J. GROMACS: fast, flexible, and free. Journal of computational chemistry 2005, 26 (16), 1701-1718.

53. Abraham, M. J.; Murtola, T.; Schulz, R.; Páll, S.; Smith, J. C.; Hess, B.; Lindahl, E. J. S. GROMACS: High performance molecular simulations through multi-level parallelism from laptops to supercomputers. 2015, 1, 19-25.

54. Palumbiny, C. M.; Liu, F.; Russell, T. P.; Hexemer, A.; Wang, C.; Müller-Buschbaum, P. The crystallization of PEDOT: PSS polymeric electrodes probed in situ during printing. Advanced materials 2015, 27 (22), 3391-3397.

55. Yesylevskyy, S. O.; Schäfer, L. V.; Sengupta, D.; Marrink, S. J. Polarizable water model for the coarse-grained MARTINI force field. PLoS Comput Biol 2010, 6 (6), e1000810.

56. Modarresi, M.; Franco-Gonzalez, J. F.; Zozoulenko, I. Morphology and ion diffusion in PEDOT: Tos. A coarse grained molecular dynamics simulation. Physical Chemistry Chemical Physics 2018, 20 (25), 17188-17198.

57. Vögele, M.; Holm, C.; Smiatek, J. Coarse-grained simulations of polyelectrolyte complexes: MARTINI models for poly (styrene sulfonate) and poly (diallyldimethylammonium). The Journal of chemical physics 2015, 143 (24), 243151. 
58.

Rolland, N.; Modarresi, M.; Franco-Gonzalez, J. F.; Zozoulenko, I. Large scale mobility calculations in PEDOT (Poly (3, 4-ethylenedioxythiophene)): Backmapping the coarse-grained MARTINI morphology. Computational Materials Science 2020, 179, 109678.

59. $\quad$ Rehmen, J.; Zuber, K.; Modarresi, M.; Kim, D.; Charrault, E.; Jannasch, P.; Zozoulenko, I.; Evans, D.; Karlsson, C. Structural Control of Charge Storage Capacity to Achieve 100\% Doping in Vapor Phase-Polymerized PEDOT/Tosylate. ACS omega 2019, 4 (26), 21818-21826.

$60 . \quad$ Berendsen, H. J.; Postma, J. v.; van Gunsteren, W. F.; DiNola, A.; Haak, J. R. Molecular dynamics with coupling to an external bath. The Journal of chemical physics 1984, 81 (8), 3684-3690.

61. Alessandri, R.; Uusitalo, J. J.; de Vries, A. H.; Havenith, R. W.; Marrink, S. J. Bulk heterojunction morphologies with atomistic resolution from coarse-grain solvent evaporation simulations. Journal of the American Chemical Society 2017, 139 (10), 3697-3705.

62. Gobbo, C.; Beurroies, I.; de Ridder, D.; Eelkema, R.; Marrink, S. J.; De Feyter, S.; van Esch, J. H.; de Vries, A. H. MARTINI model for physisorption of organic molecules on graphite. The Journal of Physical Chemistry C 2013, 117 (30), 15623-15631.

63. Franco-Gonzalez, J. F.; Rolland, N.; Zozoulenko, I. V. Substrate-Dependent Morphology and Its Effect on Electrical Mobility of Doped Poly (3, 4-ethylenedioxythiophene)(PEDOT) Thin Films. ACS applied materials \& interfaces 2018, 10 (34), 29115-29126.

64. Bussi, G.; Donadio, D.; Parrinello, M. Canonical sampling through velocity rescaling. The Journal of chemical physics 2007, 126 (1), 014101.

65. Essmann, U.; Perera, L.; Berkowitz, M. L.; Darden, T.; Lee, H.; Pedersen, L. G. A smooth particle mesh Ewald method. The Journal of chemical physics 1995, 103 (19), 8577-8593.

66. Skånberg, R.; Linares, M.; König, C.; Norman, P.; Jönsson, D.; Hotz, I.; Ynnerman, A. In VIA-MD: visual interactive analysis of molecular dynamics, Workshop on Molecular Graphics and Visual Analysis of Molecular Data, 2018; 2018; pp 19-27.

67. Eisenhaber, F.; Lijnzaad, P.; Argos, P.; Sander, C.; Scharf, M. The double cubic lattice method: efficient approaches to numerical integration of surface area and volume and to dot surface contouring of molecular assemblies. Journal of Computational Chemistry 1995, 16 (3), 273-284.

68. Sahalianov, I.; Hynynen, J.; Barlow, S.; Marder, S. R.; Müller, C.; Zozoulenko, I. UV-to-IR Absorption of Molecularly p-Doped Polythiophenes with Alkyl and Oligoether Side Chains: Experiment and Interpretation Based on Density Functional Theory. The Journal of Physical Chemistry B 2020, 124 (49), 11280-11293. 